\title{
La oferta de recursos y materiales de apoyo a la enseñanza y el aprendizaje a través de la web
}

\author{
Concepción María Jiménez Fernández \\ Universidad Internacional de La Rioja - UNIR, España
}

CASE REPORTS

\section{Resumen}

Objetivo. En este artículo se describe el análisis llevado a cabo sobre las 517 webs de biblioteca escolar de centros de educación primaria existentes en las diez provincias que conforman dos comunidades autónomas cercanas, Andalucía y Extremadura, con miras a conocer el diagnóstico de la situación en lo que se refiere a la oferta de recursos y materiales de apoyo a la enseñanza y el aprendizaje a través de la web. Método. Se justifica la elección de estas dos comunidades autónomas así como el procedimiento seguido en el diseño de la investigación, no experimental, cuantitativa y aplicada. Resultados/conclusiones. Los resultados del análisis revelan que ninguna biblioteca escolar de las estudiadas supera el $25 \%$ de webs que aportan materiales y recursos para el profesorado y el alumando. Se finaliza con la exposición de las conclusiones del estudio y con la propuesta de creación de una web modelo extrapolable a cualquier biblioteca escolar de educación primaria en España.

Palabras clave

Biblioteca escolar ; Tecnología de la información ; Aprendizaje ; Nuevas tecnologías ; Medios de enseñanza

\section{The supply of resources and materials to support teaching and learning through the web}

\section{Abstract}

Objetive. In this article there is described the analysis carried out on 517 school library webs of primary education centers in ten provinces that shape two nearby autonomous communities, Andalusia and Estremadura, with a view to knowing the diagnosis of the situation regarding the offer of teaching and learning support resources and materials through the web. Method. It is justified these two autonomous communities choice as well as the procedure followed in the investigation design, not experimental, quantitative and applied.Results/conclusions. The analysis results are also presented, which reveal that no school library of the studied ones overcomes $25 \%$ of webs that contribute materials and resources for the teachers and the students. It concludes with the exhibition of the study conclusions and with a web model proposal that can be extrapolated to any school library of primary education of Spain.

\section{Keywords}

School Library ; Information Technology ; Learning ; New Technologies ; Teaching Aid 


\section{Introducción}

La organización de un centro educativo está estrechamente ligada a la indispensable existencia de una biblioteca escolar, una biblioteca inserta en la escuela. Pero esa biblioteca, al igual que la escuela, debe adaptarse a las transformaciones que hoy por hoy se están produciendo en el ámbito de la educación gracias, sobre todo, a los cambios sociales y tecnológicos en los que estamos sumergidos. Así se ratifica en el documento Bibliotecas escolares "entre comillas" de Miret et al. (2010, p. 17) cuando se afirma que la escuela está inmersa en una profunda transformación y ha de renovar una serie de tradiciones asentadas que afectan a los contenidos de la enseñanza, a los métodos y a los recursos que la hacen posible. Es decir, una escuela que ya no se basa en el libro de texto sino que "enseñe y organice su currículo pensando en mostrar habilidades necesarias entre los alumnos que favorezcan el aprendizaje, y en este escenario la biblioteca escolar jugaría un papel imprescindible. La escuela se debe adaptar a los cambios, no ser la escuela de siempre" (Durban Roca (2010, p. 45). La biblioteca escolar se constituye entonces como un entorno de primer orden para aportar desde la escuela respuestas a las demandas de la sociedad de la información y del conocimiento. El papel de la biblioteca es relevante a la hora de acceder de forma crítica a la información y de crear conocimientos, es decir, que va más allá del simple ofrecimiento de servicios bibliotecarios y de diferentes actividades.

Pero disponer de una sala repleta de libros y otros materiales en un centro educativo -como suele ocurrir en la mayoría de los casos-, no significa tener una biblioteca escolar, al igual que no se debería entender que por el mero hecho de contar con un espacio dedicado a biblioteca estaría justificada su existencia. Cillanueva et al. (2004, p. 18), en su artículo sobre la importancia de la biblioteca escolar, analizan la situación en la que se encuentran las bibliotecas escolares y apuntan que "con frecuencia, aunque no siempre es así, la biblioteca escolar se justifica en un centro cuando este cuenta con una habitación sombría cuya llave nunca se sabe dónde está, en la que se encierran unos cuantos libros añejos en estanterías desvencijadas". Por tanto, se reafirma que no es suficiente poder disponer de un espacio dedicado a biblioteca sino que es imprescindible conocer el para qué se dispone, por lo que debe ser útil, no solo para el alumnado sino también para el profesorado (Durban Roca 2009).

De acuerdo con estas premisas, una biblioteca en la escuela solo estaría justificada cuando la organización y las actividades que realiza se enfocan desde una perspectiva curricular, es decir, en relación con los aprendizajes que se llevan a cabo en la escuela (López Fernández 1997). La biblioteca escolar, así entendida, se transformaría en un verdadero elemento nuclear en los diferentes procesos de enseñanza y aprendizaje, aquella que tiene entre sus diferentes funciones la de convertirse en una puerta de acceso al conocimiento con fines educativos y documentales. No olvidemos que en la sociedad actual el alumno ya no es considerado un mero consumidor de información sino que debe saber cómo aprender a aprender, a investigar, y la biblioteca se ha de adaptar a esas necesidades.

Del mismo modo, los servicios de la biblioteca escolar también han de adaptarse a esos cambios que se están produciendo en la sociedad. Uno de los principales sería la integración de las tecnologías en la educación para así hacer posible la formación a lo largo de toda la vida. De acuerdo con ello, Rodríguez Junco (2010, p. 34) expone que, entre los servicios obligatorios de una biblioteca escolar del futuro -y también del presente- está el de convertirse en centro de servicios multidisciplinares aliado, en todo momento, del sistema educativo.

Pero, para estar realmente al servicio del usuario, es imprescindible que todos puedan acceder a la biblioteca en cualquier momento y desde cualquier lugar. Esto se lograría más fácilmente gracias a las tecnologías y en concreto a la web de la biblioteca escolar, que ha de ser considerada como un servicio más que la biblioteca escolar presta a sus usuarios. Así lo estima Aurora Cuevas cuando explica que las posibilidades informativas y comunicativas que presenta la red $-\mathrm{y}$, por ende, la web- podría hacer posible una escuela diferente, más cercana a la realidad del alumno, abierta al mundo, una escuela en la que alumnos y profesores puedan acceder a materiales didácticos de todo tipo y a un entorno informativo de una riqueza sin precedente que favorezcan la construcción del conocimiento. Para aprovechar estas posibilidades se necesitan infraestructuras complementarias en las bibliotecas escolares, un medio privilegiado con capacidad para albergar recursos impresos y digitales en igualdad de condiciones en el que los alumnos puedan continuar sus aprendizajes, iniciar otros nuevos y disfrutar del ocio (Cuevas Cerveró 2007, p. 72). La importancia de la web en la biblioteca escolar queda también recogida claramente en el Plan de Lectura y Bibliotecas Escolares de la Junta de Andalucía (2006) donde se afirma que "puesto que la presencia de los centros en Internet es y será cada vez más evidente y como la biblioteca debe tener un papel relevante en el quehacer educativo, es necesario que esta tenga una presencia concreta y destacada en la web". 
Pero, ¿qué es una página web? Según Merlo se entiende "como el documento escrito en un lenguaje de marcado, con una localización única dentro de un servidor" (Merlo Vega 2009, p. 317). Sin embargo, cualquier web puede ser utilizada en un momento determinado como medio para llevar a cabo ciertos aprendizajes, pero referidas al mundo de la educación se puede afirmar que "webs de interés educativo son solamente aquellos que tienen una clara utilidad en algún ámbito del mundo educativo" (Romagnoli et al. 2001, p. 107). También, dentro del ámbito educativo, existen diferentes clasificaciones de una web de acuerdo con las funciones que realizan: proporcionar información de todo tipo, ya sea textual, gráfica, auditiva o audiovisual; facilitar la obtención de materiales educativos en línea; posibilitar la comunicación con otras personas; facilitar la realización de aprendizajes en línea; facilitar gestiones administrativas; actuar como medio publicitario; así como entretener y motivar.

De esa manera, en este trabajo se mostrará un diagnóstico de cómo las webs de biblioteca escolar de centros educativos de primaria de Andalucía y Extremadura se convierten en un aliado para facilitar el acceso a otras fuentes que sirvan de complemento y apoyo a la labor de enseñanza y aprendizaje. Además, se presenta un modelo de web con contenidos útiles para el profesorado y los alumnos.

\section{Objetivos y metodología aplicada}

No se debe olvidar que el concepto de biblioteca escolar hoy en día hace referencia no solo a materiales impresos como libros o revistas, sino también a un centro de recursos para el aprendizaje que también incluye materiales audiovisuales, internet, recursos electrónicos y digitales. La exclusividad de la palabra impresa y el libro como objeto estrella de la biblioteca ha dejado de ser una constante desde hace años. Ahora se da cabida a nuevos soportes y nuevos medios de información y comunicación. Al hilo de esta idea, en el Plan de trabajo y autoevaluación de la biblioteca escolar (2011, p. 18), se dice que la biblioteca escolar ha de garantizar el acceso a la información en distintos soportes y formatos, tanto para el regocijo como para satisfacer el deseo de aprender e investigar. El principal objetivo de este estudio es pues comprobar cómo se oferta este tipo de recursos previamente seleccionados a través de las webs de biblioteca escolar. Concretando un poco más, se pretende examinar qué información se proporciona a profesores y alumnos desde las webs de las bibliotecas escolares de centros de primaria de Andalucía y Extremadura con fines formativos e informativos. A partir de esos resultados se propone el diseño de una web que contenga este tipo de materiales y que sea aplicable a cualquier centro de primaria.

Para la realización del análisis se han tomado como referencia los centros educativos de primaria ya que es en esta etapa donde el niño "entra en contacto por primera vez con la palabra escrita -y no escrita-, con los distintos soportes documentales y con una institución que le va a proporcionar las herramientas para reconocer sus necesidades informativas, localizar los documentos precisos, encontrar la información con la que satisfacer dichas necesidades y reelaborar dicha información para transformarla en conocimiento útil para su vida" (Camacho Espinosa y Ortiz-Repiso Jiménez 2004, p. 115). Al mismo tiempo, y dado que, como ya se ha comentado más arriba, las bibliotecas escolares, por lo general, se encuentran en un estado carencial dentro de la escuela, se ha considerado conveniente estudiar comparativamente la situación de las webs de biblioteca escolar de todas y cada una de las diez provincias que forman las dos comunidades autónomas ya mencionadas. Estas dos comunidades, Andalucía y Extremadura, además de su cercanía geográfica, son pioneras en el uso de las TIC en educación (Cervero Guerrero 2009). De esa forma se pretende lograr una visión general y ciertamente real de los principales aspectos relacionados con los recursos y materiales que se ofrecen a través de la web con fines formativos e informativos de apoyo al proceso de enseñanza-aprendizaje.

Para elaborar las listas de los centros educativos de primaria existentes en Andalucía y Extremadura, así como averiguar cuáles de ellos contaban con web de biblioteca escolar, se procedió a la consulta de diferentes fuentes de información como portales, directorios, webs institucionales y guías de servicios educativos tanto nacionales como regionales (Andalucía y Extremadura) o provinciales. Una vez localizada la información que se buscaba, se comprobó que de los 3.042 centros de primaria existentes en las dos comunidades autónomas, 517 cuentan con web de biblioteca escolar. 
Tabla 1 - Número total de centros de Andalucía y Extremadura con web de biblioteca escolar

\begin{tabular}{|c|c|c|}
\hline & № de centros & Con web de biblioteca \\
\hline Almería & 224 & 21 \\
\hline Cádiz & 364 & 43 \\
\hline Córdoba & 266 & 54 \\
\hline Granada & 329 & 49 \\
\hline Huelva & 167 & 20 \\
\hline Jaén & 247 & 29 \\
\hline Málaga & 448 & 112 \\
\hline Sevilla & 513 & 91 \\
\hline Andalucía & 2558 & 419 \\
\hline Badajoz & 281 & 63 \\
\hline Cáceres & 203 & 35 \\
\hline Extremadura & 484 & 98 \\
\hline Total & 3042 & 517 \\
\hline
\end{tabular}

Para hacer el estudio se han consultado todas y cada una de esas 517 webs, es decir, el total de la población. Con el propósito de cumplir con los objetivos del estudio, se desarrolló un diseño de investigación no experimental cuantitativo, es decir, se observa una situación ya existente sin posibilidad de inferir sobre las variables. En palabras de Busha y Harter (1990, p. 153), el término observación "se usa para indicar que el objeto o tema de una investigación está siendo sometido a una estrecha vigilancia -generalmente visual- y que la información obtenida se relacionará después con proposiciones o teorías más generales". En este sentido, esta investigación es sistemática y empírica. Que sea sistemática implica que hay una disciplina para realizar la investigación científica y que no se dejan los hechos a la casualidad. Que sea empírica denota que se recolectan y analizan datos.

Al mismo tiempo, según su finalidad, y como afirman Hernández Sampieri et al. (2010, p. XXVII), una investigación puede cumplir dos propósitos fundamentales: ser una investigación básica -si produce conocimiento y teorías- y ser una investigación aplicada -si resuelve problemas o descubre nuevos conocimientos con un uso inmediato-. Según esto, se puede definir la presente investigación, en cuanto a su orientación, como aplicada ya que, a partir del análisis de las webs de biblioteca escolar de los centros de primaria de Andalucía y Extremadura, se persigue proponer unos contenidos que sirvan de modelo para el diseño y la creación de una web de biblioteca escolar. En este sentido, se busca una aplicación inmediata y directa del conocimiento obtenido.

\section{Resultados y discusión}

Si a lo largo de este artículo se ha incidido en la necesidad de integrar la biblioteca escolar en el currículo y la práctica educativa, es imprescindible otorgarle a este aspecto una posición de recurso-eje pedagógico que dé apoyo a la labor docente. De esta forma, la utilidad de la biblioteca escolar sería incuestionable y puede ser plenamente reconocida y argumentada, puesto que si se vinculase la existencia de la biblioteca solo a los materiales que esta proporciona como centro de recursos, en poco tiempo podremos podríamos prescindir de ella (Durban Roca 2010, p. 96). 
La web de la biblioteca escolar debe contar con un espacio para compartir información sobre temas que se imparten en clase y que complementen proyectos de trabajo del profesorado y del alumnado. Serían recursos y documentos que satisfacen las demandas y necesidades docentes y alumnos surgidas en las diferentes áreas curriculares. Se han valorado, por tanto, aquellos materiales vinculados con la actividad formativa e informativa de interés para la docencia y el aprendizaje.

En el primer caso, materiales de apoyo a la actividad formativa, se incluyen los periódicos y revistas profesionales; legislación; bases de datos de apoyo a la docencia donde poder buscar o adquirir documentación actualizada para realizar documentos profesionales; actividades relacionadas con proyectos y unidades didácticas realizadas por el centro; recursos impresos o electrónicos relacionados con un tema determinado; o documentación relacionada con la habilidades para investigar e informar.

En cuanto a los materiales de apoyo a la actividad informativa se han valorado aquellos que ofrezcan recursos bibliográficos y digitales sobre aspectos de interés pero no tan estrechamente relacionados con el trabajo en el aula, aunque sí pueden servir de complemento al currículo. Se incluye información sobre la localidad donde se encuentra el centro; directrices para animar a leer en clase; o portales y recursos educativos.

Se valoran, además, los recursos e ideas dirigidos al profesorado para dinamizar y fomentar el gusto por la lectura entre sus alumnos: lecturas en voz alta en clase por parte del maestro, visitas colectivas a la biblioteca para consultar información, etc. Estas acciones no se pueden realizar de forma aislada sino que deben ser consecuencia del plan pedagógico de la biblioteca integrado en el proyecto educativo y curricular del centro. Para ello el profesor debe estar involucrado y convencerse de que en clase se pueden utilizar otros materiales y medios didácticos para impartir una enseñanza activa -diferentes a los libros de texto- que les puede suministrar la biblioteca escolar, algo que no suele ocurrir.

Se considera importante que la biblioteca ofrezca este tipo de recursos formativos e informativos para profesorado con el fin, además, de que el alumnado perciba que también la biblioteca es utilizada y sirve a los adultos, a sus maestros. Un ejemplo este muy positivo para alumnos de primaria.

La información suele aparecer con una terminología diferente en cada web, pero por lo general son frases o palabras claramente descriptivas. Algunos ejemplos pueden ser: Recursos de apoyo, Recursos para el aula, Enlaces educativos de interés, etc.

Los recursos ofrecidos se presentan en la web de diferentes formas pero por lo general aparecen organizados por categorías o bien según las materias ajustándose al sistema de clasificación decimal universal (CDU).

De acuerdo con estos planteamientos y detallando esos aspectos según cada provincia, los resultados obtenidos se pueden observar en la figura siguiente:

\section{Materiales y recursos de apoyo a la docencia y el aprendizaje por provincias (\%)}

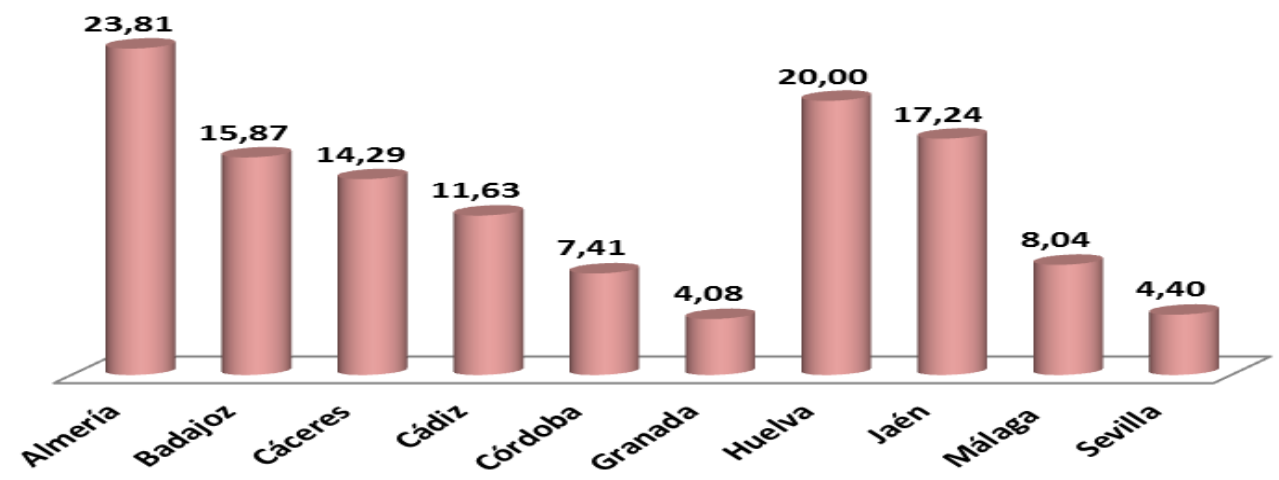

Figura 1 - Cumplimiento de los aspectos relacionados con la docencia y el aprendizaje según cada provincia 
Almería es la provincia que destaca en porcentaje de materiales de apoyo a la docencia y el aprendizaje con casi una cuarta parte $(23,81 \%)$ de las bibliotecas escolares en centros de educación primaria que contemplan este aspecto en sus webs. Con un escaso 4,08 \% es Granada la provincia que menor porcentaje tiene en este punto.

Lo más llamativo de este gráfico es que ninguna de las diez provincias supera el $25 \%$ de webs que aportan esos materiales y recursos. Este dato pone de relevancia la posible inexistencia en los centros educativos de primaria de un plan integrado de lectura y biblioteca del centro que incluya ese aspecto como uno de los más importantes para posibilitar la formación del profesorado y del alumnado, así como para facilitar la labor en el aula.

Lo que además queda de manifiesto es que la mayoría de las provincias no llegan al 20 \% de webs que ofrezcan este tipo de materiales. Este aspecto, por tanto, es uno de los menos valorados por parte de las bibliotecas escolares, lo que implica que el profesor sigue trabajando de forma aislada en el aula ajustándose al libro de texto y sin poder considerar a la biblioteca como un recurso imprescindible -que, además, serviría de ejemplo a los alumnos- para el desempeño de su labor docente.

Por otra parte, es muy común asociar el contenido de la biblioteca escolar solamente con el área de Lengua y Literatura, por lo que muchos profesores y alumnos no son conscientes de las posibilidades que una biblioteca bien gestionada y organizada puede ofrecerles para el desarrollo de su trabajo en el aula. Pero, por lo general, al frente de la biblioteca escolar suele estar un profesor que cubre de esta forma sus horas libres o que solo la abre durante el recreo para que los niños que voluntariamente quieran ir puedan sacar un libro en préstamo. La inexistencia de un bibliotecario a tiempo completo y preocupado por satisfacer las necesidades informativas, formativas y de recreo de sus usuarios implica, entre otras muchas cosas, que no se puedan aportar estos materiales de apoyo sobre las distintas áreas.

\section{Conclusiones}

Tras el análisis de los resultados expuestos en el apartado anterior, se presentan las diferentes conclusiones obtenidas en el estudio y que suponen un diagnóstico objetivo de la situación real de las webs de biblioteca escolar de centros de primaria de Andalucía y Extremadura en cuanto a su capacidad para ofrecer recursos útiles para docentes y alumnos dentro del proceso de enseñanza-aprendizaje.

Las webs de biblioteca escolar no se suelen considerar como recurso imprescindible para "aprender a aprender" en el colegio. Todavía se detecta la existencia de una enseñanza pasiva en el aula donde se recibe y digiere lo que el maestro imparte en clase y que sirve para aprobar como objetivo final. Se debería exigir la implantación de este tipo de servicio que supone un valor añadido en la labor de enseñanza y aprendizaje.

Se comprueba que las bibliotecas escolares todavía siguen siendo espacios utilizados por profesores y alumnos como lugar donde solo se accede a libros, por lo general de ficción, o como un lugar para el estudio. No se percibe como recurso indispensable de apoyo a la labor en el aula.

Queda reflejada la inexistencia, por lo general, de la figura del bibliotecario escolar en la mayoría de los centros educativos así como un plan de biblioteca integrado en el que todo el claustro esté implicado. Esto provoca que la biblioteca escolar no se adapte adecuadamente a los nuevos entornos tecnológicos con miras a satisfacer las necesidades de sus usuarios. Sería conveniente contar con el compromiso, por parte de todos los implicados en el centro educativo, no solo de crear una web de biblioteca sino de actualizarla y mantenerla con el fin de servir adecuadamente al usuario.

La biblioteca, por tanto, sigue funcionando como centro conservador y difusor de aquellos materiales que se han ido incorporando gracias a diferentes políticas de adquisiciones sin hacer uso de las posibilidades que ofrecen las tecnologías. Sería necesario que se incorporaran estas potencialidades, en concreto de la web de biblioteca escolar, y evitar el aislamiento al que todavía están sometidas muchas bibliotecas escolares.

Se evidencia el escaso interés que tiene la mayoría de los centros de todas las provincias estudiadas por ofertar recursos que, sobre todo en primaria, servirían para que el alumnado tomara mayor contacto con la lectura, la escritura y las bibliotecas y así valorar el uso y utilidad de cualquier biblioteca durante toda su vida. 


\section{Prospectiva: diseño de contenidos para enseñanza y aprendizaje en una web de biblioteca escolar}

Este estudio está basado en la investigación empírica realizada en todas las provincias que conforman las comunidades autónomas de Andalucía y Extremadura. Si se decidiese salir de estas dos comunidades, se observaría que la propuesta de contenidos sobre recursos útiles para la enseñanza y el aprendizaje que se exponen a continuación podría ser extrapolable a cualquier comunidad autónoma española por la muestra tan amplia que se ha manejado.

A través de la web de biblioteca escolar se pueden proporcionar materiales relacionados con temas que se imparten en el aula. Estos materiales pueden ser impresos, electrónicos o digitales a los que se puede acceder desde cualquier lugar y en cualquier momento.

Se incluirían novedades que pueden ir apareciendo sobre las diferentes especialidades así como noticias relacionadas con las áreas que se imparten en clase. Estas novedades también se pueden difundir mediante envíos periódicos al profesor a través del correo electrónico.

Otros tipos de información que se podrían incluir en la web de biblioteca escolar serían los siguientes:

- Recursos gráficos y audiovisuales para facilitar el estudio y asimilación de las diferentes materias.

- Directrices para motivar a los alumnos hacia el estudio así como otros materiales sobre innovaciones pedagógicas y didácticas.

- Enlaces sobre experiencias exitosas llevadas a cabo en otros centros educativos que puedan servir de ejemplo de buenas prácticas en el aula.

- Recursos sobre días especiales que se celebren a lo largo del año y que se puedan aprovechar para completar cualquier materia, así como enlaces de fomento de la lectura tanto para profesores como para alumnos.

\section{Referencias}

Busha, Charles H., Harter, Stephen P. (1990). Métodos de investigación en bibliotecología : técnicas e interpretación. México: Universidad Nacional Autónoma de México.

Camacho Espinosa, J. A., Ortiz-Repiso Jiménez, V. (2004). "Bibliotecas públicas y bibliotecas escolares : ¿colaboración, cooperación o integración en una red conjunta? Realidad y propuesta para la Comunidad de CastillaLa Mancha" En La Biblioteca Pública : compromiso de futuro, Congreso Nacional de Bibliotecas Públicas, Salamanca 17, 18 y 19 de noviembre de 2004. Ministerio de Cultura, Madrid, pp. 112-120.

Cervero Guerrero, José. (2009) Proyecto de innovación educativa, [en línea]. Disponible en internet: http://sites.google.com/site/fuentenuevaes/planes-y-proyectos-educativos/proyecto-de-innovacion-educativa

Cillanueva, T., Hortelano, L., García Ruiz, J. M. (2004). “La importancia de la biblioteca escolar”, Escuela Española, n. 3.621, pp.18-21.

Cuevas Cerveró, A. (2007). Lectura, alfabetización en información y biblioteca escolar. Gijón: Trea.

Durban Roca, Glòria. (2009). "Tensiones y distensiones. Reflexión en torno a la situación actual de la biblioteca escolar", Libro Abierto : Bibliotecas escolares de la provincia de Málaga : Boletín de información y apoyo, ํㅡ 35, febrero, pp. 6-11.

Durban Roca, Glòria. (2010). La biblioteca escolar, hoy : un recurso estratégico para el centro. Barcelona: Graó. 
Hernández Sampieri, R., Fernández-Collado, C., Baptista Lucio, P. (2010). Metodología de la investigación, 5ā ed. México: Mc Graw Hill.

López Fernández, Juan A. (1997). "La biblioteca escolar en la Región de Murcia”, Boletín de la ANABAD, t. 47, n. 2, pp. 59-88.

Merlo Vega, J.A. (2009). Información y referencia en entornos digitales: desarrollo de servicios bibliotecarios de consulta. Murcia: Universidad de Murcia.

Miret, I., Baró, M., Mañá, T., Vellosillo, I., Montero, I. 2010, Bibliotecas escolares «entre comillas», Fundación Germán Sánchez Ruipérez : Ministerio de Educación, Madrid.

Plan de Lectura y de Bibliotecas escolares en los centros educativos de Andalucía. (2006). Málaga: Junta de Andalucía, Consejería de Educación.

Plan de trabajo y autoevaluación de la biblioteca escolar, Documento de Referencia para bibliotecas escolares DR1/BECREA. 2011, Junta de Andalicía, Consejería de Educación, Málaga, [en línea]. Disponible en internet: http://www.juntadeandalucia.es/averroes/bibliotecaescolar/images/MisPdf/DR1/DR1BECREA.pdf

Rodríguez Junco, Fernanda. (2010). ¿Tienen futuro las bibliotecas? En Las bibliotecas escolares : ¿el último reino de papel?, Manuel Ortíz Cruz, Manuel Area Moreira (coords.), Consejería de Educación, Universidades, Cultura y Deportes del Gobierno de Canarias, Dirección General de Ordenación e Innovación Educativa, Centro del Profesorado Valle de la Orotava, Tenerife, pp. 27-38. Disponible también en internet: http://issuu.com/morrison46/docs/ el Itimo reino de papel [Fecha de acceso 12 de julio de 2011].

Romagnoli, C., Femenías, G., Conte, P. (2001). Internet, un nuevo recurso para la educación. Material de apoyo para profesores. Santiago de Chile: Ministerio de Educación.

\section{Datos de la autora}

Concepción María Jiménez Fernández

Doctora en Documentación, es profesora de la Universidad Internacional de la Rioja (UNIR), responsable de la biblioteca municipal de Azuaga (Badajoz) y directora de la revista Mi biblioteca.

redaccion@mibiblioteca.org

$\begin{array}{ll}\text { Recibido-Received } & : 2013-08-01 \\ \text { Aceptado-Accepted } & : 2013-12-30\end{array}$

\section{(cc) $\mathbf{E Y}$}

This work is licensed under a Creative Commons Attribution 4.0 United States License.

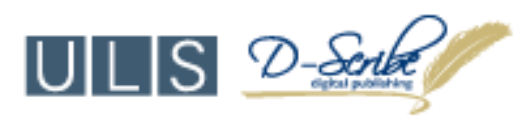

This journal is published by the University Library System of the University of Pittsburgh as part of its $\underline{D}$-Scribe Digital Publishing Program and is cosponsored by the University of Pittsburgh Press. 